\title{
Expansion of the genetic diversity of common spring wheat for resistance to biotic stress factors at the Chelyabinsk Research Institute of Agriculture
}

\author{
Igor' Kushnirenko ${ }^{1}$, Ekaterina Shreyder ${ }^{1,}$, ,Ekaterina Shaydayuk $^{2}$, Nadezhda Kovalenko ${ }^{2}$, \\ and Elena Gultyaeva ${ }^{2}$ \\ ${ }^{1}$ Chelyabinsk Scientific Research Institute of Agriculture, Russia \\ ${ }^{2}$ All Russian Institute of Plant Protection, 196608 Saint Petersburg, Russia
}

\begin{abstract}
The Chelyabinsk Research Institute of Agriculture (CRIA) conducts targeted breeding for the complex resistance of soft spring wheat to biotic and abiotic stress factors, with the involvement of alien translocations donors. At the same time, the virulence of the pathogen populations is monitored. The purpose of these studies is to study disease resistance and to characterize economically valuable traits in 60 introgressive lines created in the CRIA, and to assess their influence on the variability of pathogens populations for virulence. Using molecular markers, the high genetic diversity of the studied collection of introgressive breeding lines for effective alien resistance genes was confirmed. According to the field and laboratory studies, promising lines were identified (Lutescens (Lut.) 26708, Erythrospermum (Erythr.) 26725, Ferrugineum (Ferr.) 26727, Ferr. 26752, Ferr. 26757, Erythr. 26762, Erythr. 26775, Lut. 26720) These lines combine multipathogen diseases resistance with a complex of economically valuable characteristics (yield, grain quality). There was no effect of introgression lines of spring wheat on the variability of the pathogen population for virulence.
\end{abstract}

\section{Introduction}

Genetic protection of wheat against diseases is an important basic element of resourcesaving and environmentally friendly technologies for grain production. Its high level is achieved by cultivating varieties that are genetically diverse in terms of resistance to biotic and abiotic stress factors and their rationally placing in agroclimatic regions. Timely change of genetically resistant varieties allows to stabilize the population composition of phytopathogens and reduce the probability of epiphytotic diseases. The development of molecular markers of resistance genes allows their successful use in breeding work in all countries [1-2]. The genetic protection of wheat from rust and powdery mildew is widely used in the North American continent, in Australia and in a number of European countries [3].

*Corresponding author: shreyder11@mail.ru 
Currently, the use of the intraspecific genetic potential of soft wheat does not provide a sufficient degree of protection against diseases. In addition, the phytopathological situation is deteriorating due to the shift in the distribution area of a number of soft wheat pathogens, due to global climate change, in particular, widespread warming.

Selection for disease resistance should be carried out continuously, because parasitic microorganisms evolve together with the host and many genes that control this trait are currently not effective. In this way, the widespread use of the available limited set of known genes of soft wheat resistance to pathogens, in particular to leaf and stem rust, has led to a rapid loss of their relevance, due to the emergence of more virulent highly adapted pathotypes of these diseases pathogens [4-6].

Spring wheat in the Southern Urals is the leading grain crop, occupying up to 840 thousand hectares ( $45 \%$ in the structure of the region sown area). At the same time, grain production in the Southern Urals remains unstable, which is largely due to natural causes. In the region, drought prevails among abiotic stresses, and among biotic ones - strong annual lesion of wheat with brown rust, harmfulness of which can reach $37 \%$ [7]. In recent years, there has been an increase in the development of stem rust and spotting types.

Since 1990, the Chelyabinsk Research Institute of Agriculture has been conducting targeted selection for complex resistance to a set of stress factors, with the involvement of donors of alien translocations and resistance genes. In hybridization, donors of alien translocations from Aegilops umbellulata, Aegilops speltoides, Secale sereale, Agropyron elongatum, Ag. intermedium, Ae. tauschii, Triticum ventricosum, etc. are widely used. As a result, an extensive selection material has been created, which in the near future may be an alternative to the modern wheat assortment in the Southern Urals. It was important to determine their immunological value (group resistance to the main pathogenic complex), to characterize economically valuable traits and genetic control of disease resistance, since it is known that some alien translocations affect the quality characteristics of the created varieties. In addition, genetic management of diseases can not be carried out without information about the possible influence of new varieties on the variability of pathogen populations, and therefore, the assessment of the effect of new genotypes on populations changes of a wide group of pathogens is relevant. A comparison of the racial composition of pathogens on genetically determined breeding lines with races composition on widely grown varieties in the Ural region will make it possible to predict the influence of the new wheat material on the possible phytosanitary situation.

The aim of this research was to study the diseases resistance and complex agronomic traits in new soft spring wheat breeding lines, created at the Chelyabinsk Research Institute of Agriculture with the involving of alien translocations and to evaluate its possible influence on the formation of pathogens populations in the Southern Urals.

\section{Materials and methods}

The study included 60 wheat introgressive lines obtained with the involvement of donors carrying genetic material from Ae. speltoides, Ag. elongatum, Ag. intermedium Ae.tauschii, Secale sereale.

In the field conditions at the infectious plot of the CRIA in 2019-2020, the wheat lines was evaluated for resistance to folia diseases (rust, powdery mildew, spotting) and phenotypic traits. Disease severity of leaf and stem rust was assessed according to the Peterson scale [8], leaf blotches according to the Saari and Prescott scale [9].

Standard methods [10-11] were used for estimation of economically useful parameters and evaluation of technological qualities of grain and flour.

In laboratory conditions, in the seedling stage, the resistance of breeding lines to leaf, stem and yellow rusts, tan spot and spot blotch was studied. Resistance to leaf rust was 
evaluated by using of three isolates marked by virulence to the genes $\operatorname{Lr} 9, \operatorname{Lr} 19, \operatorname{Lr} 26$ and Chelyabinsk and Krasnodar populations of Puccinia triticina; to stem rust - by inoculation of Chelyabinsk population of $P$. graminis, which was characterized by avirulence to varieties and lines with the genes $S r 24, \operatorname{Sr} 25$ and $S r 31$; to yellow rust - by the Novosibirsk and Krasnodar populations of $P$. striiformis, differ from each other by virulence.

Type of reaction to leaf rust was evaluated according to the Maines and Jackson scale, to stem rust - according to the Stackman and Levin scale, and to yellow rust - according to Gassner and W. Straib [8]. Plants with reaction type 0, 1, 2 were classified as resistant, 3, 4, $\mathrm{X}$ - as susceptible.

To study the resistance to tan spot, the races produced a different set of Pyrenophora tritici-repentis exotoxins were used. Isolates were received from the studied wheat lines. Resistance to tan spot was assessed 6-7 days after inoculation according to a 5-point scale [12]. Plants with a score of 1,2 were classified as resistant; with a score of 3 - as moderately susceptible, with a score of 4, 5 - as susceptible.

Molecular markers were used to identify 19 genes $(\operatorname{Lr} 1, \operatorname{Lr} 3 a, \operatorname{Lr} 9, \operatorname{Lr} 10, \operatorname{Lr} 19, \operatorname{Lr} 20$, Lr21, Lr24, Lr25, Lr26, Lr28, Lr29, Lr34, Lr35, Lr37, Lr41, Lr47, Lr51,LrSp) [13-15].

For genetic studies of pathogens population, leaves with urediniopustules and spots were collected from wheat studied breeding lines and commercial varieties susceptible to diseases at the field conditions. The preparation of monopustule and monoconidial isolates and the virulence analysis were carried out according to the previously described methods [16].

\section{Results and discussion}

In the field conditions of the Southern Urals in 2019-2020, more than 70\% of the studied lines were characterized by a high level of resistance to leaf and stem rust $(0 \%$ no disease symptoms). The severity of leaf rust in the field conditions in years of study varied from 0 to $70 \%$, and stem rust - from 0 to $40 \%$. More than $40 \%$ of the wheat lines showed resistance to powdery mildew, and $30 \%$ - to blotches (Septoria blotch and tan spot). Disease severity of powdery mildew and blotches was higher in 2019 (up to 40\%). According to visual symptoms, Septoria blotch was more distributive spot. Its severity on the studied lines varied from 0 to $30 \%$. According to the results of the first year of research, lines resistant to one disease were identified, as well as lines with group resistance to two or more diseases. Most of the breeding lines in the field conditions were characterized by high resistance to lodging, good phenotypic traits, as well as a fairly high ocular grain rating (from 3.9 to 4.5 scores).

When inoculating the lines by stem rust population, a resistant type of reaction $(0,1,2$ scores) was detected in all lines, with the exception of Lut. 26534. In the field conditions, disease symptoms are also noted on this line.

A similar situation was noted in the study of leaf rust. A resistance type of reaction to leaf rust (score $0.1,2$ ) was observed in $92 \%$ of the lines. The lines Lut. 26720, Lut. 26721, and Erythr. 26759 were susceptible to all clones and populations. The Lut. 26534 line showed a moderately resistant type of reaction when inoculated with a test isolate virulent to $\operatorname{Lr} 19$ and susceptible to all other isolates and populations. The Erythr. 26775 line was susceptible to a isolate virulent to $\operatorname{Lr} 26$ and the Chelyabinsk pathogen population. The Ferr. 26635 line was affected by all isolates and populations avirulent to $\operatorname{Lr} 9$ and had a susceptible reaction when infected with a virulent isolate (K9) and the Chelyabinsk population, also virulent to $\operatorname{Lr} 9$. These results suggests that it has this gene. All the abovementioned lines susceptible to leaf rust in the seedling stage were characterized by resistance in the field conditions. This indicates that they have adult plant resistance genes, 
or genes that have lost their effectiveness in single, but in combinations can provide expression of resistance in the field conditions.

Only five lines showed resistance to the Krasnodar and Novosibirsk populations of $P$. striiformis: Erythr. 26688, Erythr. 26744, Erythr. 26760, Erythr. 26763, Erythr. 26764. The Erythr. 26752 and Barbarossa 26607 lines were resistant to the Krasnodar population, but moderately susceptible to the Novosibirsk population The number of lines resistant to the causative agent of yellow rust was significantly less $(8 \%)$ than to the pathogens of leaf and stem rust.

Most of the studied breeding lines in the seedling phase showed a susceptible reaction to Septoria blotch (score 5). Only four lines were characterized by moderate resistance (score 2-4): Lut. 26708, Lut. 26714, Fer. 26758, Fer. 26774. The number of lines resistant to tan spot was significantly higher. When inoculated with the Omsk and Kazakhstan populations of $P$. tritici-repentis, 57\% of the lines showed resistant (score 0-1) and moderately resistant reactions (score 2). Similar results were obtained when inoculated by Chelyabinsk races of $P$. tritici-repentis producing different exotoxins (67\% resistant).

Using molecular markers, the high genetic diversity of the studied collection of introgressive breeding lines for highly effective alien resistance genes was confirmed. The known alien translocation from Ag. elongatum with genes $\mathrm{Lr} 19 / \mathrm{Sr} 25$ and gene $L R 24 / \mathrm{Sr} 24$, from Ae. speltoides with genes $L r S p$ and $S r S p$, from Ae. tauschii with gene $L r 21$ from $A g$. intermedium with gene Lr6Agi2, from Ae. umbellulata with gene $\operatorname{Lr} 9$, S. sereale with genes Lr26, Sr31, $\operatorname{Yr} 9, \operatorname{Pm} 8, T$. monococcum with gene $\mathrm{Sr} 35$ and noneffective genes from $T$. aestivum Lr1, Lr10, Lr34/Sr57 were identified. Many of the studied lines carried two or more alien translocations. At the same time, a number of highly resistant lines (Lut. 26706, Erythr. 26759, Erythr. 26760, Erythr. 26762, Erythr. 26763, Lut. 26765) revealed only ineffective ones $(\operatorname{Lrl}, \operatorname{Lr} 3, \operatorname{Lr} 10)$, which suggests the presence of new additional genes in their genotype.

The dominant allele Tsn1 of caused agent of tan spot was identified in three lines Lut. 26720, Lut. 26721, and Erythr. 26763. The absence of this allele in most of the other studied lines probably explains their moderate reaction to $P$. tritici-repentis isolates.

The virulence and racial composition of $P$. triticina, $P$. graminis and $P$. tritici-repentis isolates isolated from affected introgressive lines and varieties of soft spring wheat grown in the region are characterized. There were no significant differences in the racial composition of leaf and stem rust populations in 2019 and 2020. The resistance of the lines with the $S r 31$ gene to the $P$. graminis isolates indicates the absence of the potentially dangerous $\mathrm{Ug} 99$ race in the Ural population. In the analysis of the virulence of the $P$. triticirepentis population, compared to 2019, an increase in potentially dangerous races producing exotoxin ToxA was noted (42\% and 56\%).

The conducted studies of the populations of the harmful wheat complex on new introgressive lines and commercial varieties in the conditions of the Southern Urals indicate its certain stability and the absence of significant changes in virulence. Most of the resistance genes identified in introgressive lines belong to the group of highly effective ones and will be able to provide genetic management of wheat from diseases in the region in case of further production introduction of the most promising lines.

As a result of laboratory evaluation of the technological qualities of grain, lines with an increased content of protein and gluten were identified: Lut. 26708 (translocation from $\mathrm{Ag}$. intermedium); Erythr. 26725, Ferr. 26727 (Ag. elongatum); Ferr. 26752, Ferr. 26757 (Ae. speltoides), Erythr. 26762 (S. sereale); Erythr. 26775, Lut. 26720. They are also characterized by a higher level of yield, compared to standard varieties, and multipathogen disease resistance s. Only ineffective $L r$ genes $(L r 1, L r 3, L r 10)$ were detected in the resistant to rust lines Erythr. 26775, Lut. 26720, which suggests the presence of additional genes in them genotypes. 


\section{Conclusion}

As a result of the research, a high genetic diversity for disease resistance of the soft spring wheat, created in the CRIA with the involvement of alien donors, was revealed. More than $20 \%$ of breeding lines were characterized by multipathogen resistance to diseases, good phenotypic and economically valuable traits. Most of them have identified disease resistance genes that were not previously used in wheat breeding in Russia. 8 most promising lines were selected: Lut. 26708; Erythr. 26725, Fer. 26727; Fer. 26752, Fer. 26757, Erythr. 26762; Erythr. 26775, Lut. 26720. These lines combine a complex of economically valuable traits (yield, grain quality) and resistance to leaf and stem rust. The influence of promising spring wheat lines bearing alien translocations on the variability of pathogen populations by virulence was not observed, which will contribute to the stability of the genetic protection in the conditions of the Southern Urals.

The current research was supported by RFBR grant No. 19-016-00052.

\section{References}

1. P.K. Gupta, H.S. Balian, R.K. Varshney, K.S. Gill, Plant Breed, 132, 431 (2013)

2. J. Huerta-Espino, R.P. Singh, S. Germain, D. Mccallum, R.F. Park, W.Q. Chen, S.C. Bhardwaj, H. Goyeau, Euphytica, 179, 143 (2011)

3. M.D. Bolton, J.A. Kolmer, D.F. Garvin, Plant Pathol, 9, 563 (2008)

4. S.N. Sibikeev., V.A. Krupnov, Vestnik Saratovskogo gosuniversiteta im. Vavilova. Special issue, 92 (2007)

5. Z.A. Pretorius, R.P. Singh, W.W. Wagoire, T.S. Payne, Plant Dis., 84, 203 (2000)

6. R.P. Singh, D.P. Hodson, J. Huerta-Espino, Y. Jin, P. Njau, R. Wanyera, S.A. HerreraFoessel, R.W. Ward, Adv. Agron, 98, 271 (2008)

7. V.A. Tyunin, E.R. Shreider, E.I. Gultyaeva, E.L. Shaydayuk, Vavilov J Genet. Breed, 21, 523 (2017)

8. R.A. McIntosh, C.R. Wellings, R.F. Park, Wheat rusts. An atlas of resistance genes (1995)

9. E.E. Saari, J.M. Prescott, Plant Dis. Rep., 59, 377 (1975)

10. M.A. Fedin, Methodology of state variety testing of agricultural crops, technological assessment of grain, cereals and leguminous crops (1988)

11. Methodology of state variety testing of agricultural crops (1988)

12. L. Lamari, C.C. Bernier, Can. J. Plant Sci., 11, 49 (1989)

13. B.D. McCallum, C.W. Hiebert, S. Cloutier, G. Bakkeren, S.B. Rosa, D.G. Humphreys, G.F. Marais, C.A. McCartney, V. Panwar, C. Rampitsch et al, Can. J. Plant Pathol, 38, 1 (2016) https://maswheat.ucdavis.edu

14. L. Błaszczyk, I. Kramer, F. Ordon, J. Chełkowski, M. Tyrka, J. Vida, I. Karsai, Cereal Res. Comm. 36, 201 (2008) https://maswheat.ucdavis.edu

15. L.S. Vanzetti, P. Campos, M. Demichelis, L.A. Lombardo, P.R. Aurelia, L.M. Vaschetto, C.T. Bainotti, M. Helguera, Electronic Journal of Biotechnology (2011) https://maswheat.ucdavis.edu

16. E.I. Gultyaeva. N.M. Kovalenko, V.P. Shamanin, V.A. Tyunin, E.R Shreyder., E.L Shaydayuk., A.I. Morgunov, Vavilov Journal of Genetics and Breeding, 22, 363 (2018) 